\title{
LA CORRESPONDANCE D'ÉLIE BOUHÉREAU (1643-1719) : LES ANNÉES FOLÂTRES
}

\author{
Ruth Whelan
}

Armand Colin | « Littératures classiques »

2010/1 $\mathrm{N}^{\circ} 71 \mid$ pages 91 à 112

ISSN 0992-5279

ISBN 9782908728620

Article disponible en ligne à l'adresse :

https://www.cairn.info/revue-litteratures-classiques1-2010-1-page-91.htm

Distribution électronique Cairn.info pour Armand Colin.

(C) Armand Colin. Tous droits réservés pour tous pays.

La reproduction ou représentation de cet article, notamment par photocopie, n'est autorisée que dans les limites des conditions générales d'utilisation du site ou, le cas échéant, des conditions générales de la licence souscrite par votre établissement. Toute autre reproduction ou représentation, en tout ou partie, sous quelque forme et de quelque manière que ce soit, est interdite sauf accord préalable et écrit de l'éditeur, en dehors des cas prévus par la législation en vigueur en France. Il est précisé que son stockage dans une base de données est également interdit. 
Ruth Wh el an

\section{La correspondance d'Élie Bouhéreau (1643-1719) : les années folâtres}

Qui de nos jours connaît le nom d'Élie Bouhéreau, ce protestant rochelais, érudit de province, et épistolier assidu par surcroît, qui se réfugia dans les îles dites britanniques après la Révocation de l'Édit de Nantes ? Pourtant, enfouies dans les archives de la Bibliothèque Marsh à Dublin, dont Bouhéreau fut le premier bibliothécaire en titre à partir de 1701, se trouvent à peu près 1250 lettres à lui adressées entre 1661 et 1689 par plus de 150 correspondants ${ }^{1}$. Correspondance essentiellement passive, donc, que cet homme cultivé tenait à préserver de l'orage qui s'est abattu sur les protestants de France sous le règne de Louis XIV ${ }^{2}$, et qu'il a fait transporter dans un coffre-fort d'abord en Angleterre et ensuite en Irlande, et qu'il a fini par déposer à la Bibliothèque en 1714. Pourquoi emporter ces papiers avec lui, lorsqu'il s'évadait de la France avec sa famille dans des conditions hautement périlleuses, défiant les Déclarations royales qui interdisaient la sortie du royaume aux sujets de la religion réformée ${ }^{3}$ ? On sait que dans l'ancienne France,

1 Une partie minime de cette correspondance est adressée à Blandine Richard, mère de Bouhéreau, morte à Dublin en avril 1700 .

2 J'emprunte cette métaphore à Ézéchiel Spanheim, Relation de la Cour de France en 1690, éd. M. Richard, Paris, Mercure de France, 1973, p. 50. Sur Spanheim, voir R. Whelan, « Le revers de la médaille : La Relation de la Cour de France (1690) d'Ézéchiel Spanheim », dans J. Garapon (éd.), Mémoires d'État et culture politique en France (XVI -XIXe siècles), Nantes, Éditions Cécile Defaut, 2007, p. 141-150.

3 La Déclaration royale interdisant aux sujets du roi de France de quitter le territoire sans autorisation date du $1^{\text {er }}$ février 1669 et fut reprise par plusieurs Déclarations en 1681, 1685 et 1686. Voir É. Labrousse, Essai sur la Révocation de l'Édit de Nantes : une foi, une loi, un roi ?, Genève / Paris, Labor et Fides, 1985, p. 146-147. Bouhéreau a fait sortir de France son importante bibliothèque (de plus de deux mille livres) dans la valise diplomatique de William Trumball, ambassadeur d'Angleterre auprès de Louis XIV. Lui avait-il aussi confié le coffre-fort, ou l'avait-il transporté lui-même ? On ne le sait pas. Sur la bibliothèque de Bouhéreau, voir P. Benedict et P.-O. Léchot, «The library of Élie Bouhéreau : the intellectual universe of a Huguenot refugee and his family ", dans M. McCarthy et 
les particuliers gardaient chez eux dans un coffre-fort les contrats et actes notariés, les lettres et documents qui constituaient des attestations de leur état civil, de leurs biens, et de leur rang social ${ }^{4}$. Il s'agissait en somme de leurs pièces d'identité, de tout ce qui certifiait leur signalement, leur ascendance et, en somme, leurs racines ${ }^{5}$. Ainsi, il ne serait pas inintéressant d'ouvrir ce coffre-fort déposé à la Bibliothèque Marsh et d'interroger ces pièces d'identité devenues pièces d'archives, car elles auront sans doute des secrets à nous livrer sur la vie de ce réfugié rochelais qui finit ses jours bibliothécaire à Dublin.

Néanmoins, il nous est impossible de reconstituer avec exactitude la liste des documents mis en dépôt à la Bibliothèque, car le coffre-fort s'est volatilisé, emportant avec lui une partie de ce qu'il contenait. Tout ce que l'on sait de science certaine, c'est qu'en 1853, après l'avoir rouvert, on y trouva divers manuscrits parmi lesquels figuraient les archives de l'Église française de La Rochelle (restituées depuis) et treize paquets de lettres adressées à Bouhéreau, dont on signala l'existence à la Société de l'Histoire du Protestantisme Français, tout en estimant que si « une partie de cette correspondance offre de l'intérêt ; une autre est sans valeur $^{6} »$. En fait, mésestimant la valeur de ces vieux manuscrits français, T. R. W. Craddock, le bibliothécaire de l'époque, les a offerts à son assistant Robert Travers, qui les a peut-être vendus ou offerts à des particuliers, car les lettres disparaissent pendant un certain temps. En 1903, pourtant, trois paquets refont surface dans les archives personnelles de J. J. Digges La Touche, récemment décédé, et sont récupérés par la Bibliothèque ; trois années plus tard, six autres paquets resurgissent, quand Lord Iveagh, de la famille Guinness, s'apprête à les déposer dans la bibliothèque de Trinity College, à Dublin ; ils sont également restitués à la Bibliothèque Marsh en 1906. Manquent donc quatre paquets de lettres, et peut-être aussi les contrats et actes notariés que contenait probablement ce coffrefort en 1853. Il serait inutile de faire des hypothèses sur ce qui a disparu ; mieux vaut tout simplement constater que cette correspondance avait aux yeux de Bouhéreau une valeur telle qu'il a tenu à la transporter partout avec lui sur le douloureux chemin de l'exil. Ces lettres constituaient sans doute pour lui un précieux souvenir de ses origines, de ses attaches, et de cette vie qu'il menait en France avant la rupture provoquée par la Révocation.

A. Simmons (éd.), Marsh's Library. A Mirror on the World. Law, learning and libraries (1650-1750), Dublin, Four Courts Press, 2009, p. 165-184.

4 N. Zemon Davis, « Ghosts, kin, and progeny : some features of family life in early modern France », Daedalus, n 106, 1977, p. 87-114.

5 C. Lougee Chappell, « Paper memories and identity papers : why Huguenot refugees wrote memoirs ", dans B. Tribout and R. Whelan (éd.), Narrating the self in early modern Europe, Oxford, Peter Lang, 2007, p. 121-138.

6 Edward Granvelle, comte de Saint-Germans, « Mss. français conservés à la bibliothèque Marsh, à Dublin », Bulletin de la Société de l'histoire du protestantisme français, $\mathrm{n}^{\circ} 2,1853$, p. 407-410. 
Contrairement à ce que l'on pensait au $\mathrm{XIX}^{\mathrm{e}}$ siècle, les lettres conservées - pour incomplètes et lacunaires qu'elles soient - portent dans l'ensemble un témoignage d'une grande valeur sur la vie d'un jeune protestant français dans la première moitié du règne personnel de Louis XIV. Quoiqu'il nous reste très peu de lettres écrites par Bouhéreau à cette époque de sa vie, celles de ses correspondants, dont la grande majorité étaient eux aussi protestants, nous permettent de retracer, au moins dans les grandes lignes, les processus qui ont peu à peu transformé ce fils de pasteur en homme de lettres. Si la plupart des lettres proviennent de notables (pasteurs, professeurs, avocats, médecins, auteurs et érudits), un nombre non négligeable des lettres conservées sont de différents membres de la famille - côté Bouhéreau (côté paternel) aussi bien que côté Richard (côté maternel) ; ont survécu aussi des missives d'hommes d'affaires : banquiers, libraires, marchands et autres clients de la famille Bouhéreau. Ainsi un des intérêts de cette correspondance réside dans l'ampleur des connaissances qu'elle nous permet d'avoir du réseau ou plus précisément des réseaux épistolaires de Bouhéreau, et du rôle important d'intermédiaire qu'il est arrivé à jouer au sein de ces différents réseaux, grâce à sa position matérielle assez exceptionnelle pour l'époque.

Dans un deuxième temps, je me pencherai sur la pratique de la lettre qui se dégage de la correspondance de Bouhéreau, et surtout des lettres qu'il échangeait régulièrement avec ses anciens professeurs et camarades d'études, dont il avait fait la connaissance à l'Académie de Saumur. À de certaines exceptions près, ces lettres sont des lettres privées, dans le sens un peu flou qu'avait le mot au XVII ${ }^{\mathrm{e}}$ siècle, que les différents correspondants écrivaient au fil de la plume, et envoyaient aussitôt. Certes, Bouhéreau comptait parmi ses correspondants de véritables épistoliers qui rédigeaient au moins certaines de leurs lettres dans l'idée d'une publication ultérieure, je pense par exemple à Valentin Conrart, ou à Tanneguy Le Fèvre, helléniste de renom et professeur à l'Académie de Saumur, qui a publié au milieu des années 1660 des recueils d'epistolce latines dont une vingtaine adressées à Bouhéreau ${ }^{7}$. Ces deux correspondants mis à part, il ne s'agit surtout pas, dans la plupart des lettres qu'on lui écrivait, de ce style " négligé », cultivé avec art par les épistoliers de grande envergure, mais plutôt d'une véritable spontanéité dans la rédaction de la lettre. On écrivait pour rester en contact, pour demander ou rendre des services, pour communiquer des nouvelles, et parfois tout simplement pour s'épancher à cœur ouvert. Il s'agissait néanmoins de correspondants instruits pour la plupart, qui avaient fait des études de rhétorique, qui lisaient les Lettres (dont une édition paraît en 1661) de Balzac à Chapelain, qui admiraient Conrart et Ménage, et qui avaient par conséquent le sens de ce qu'une correspondance devrait être. Il est intéressant donc d'étudier ce que la lettre représentait pour eux, les liens qu'elle leur

7 Tanaquilli Fabri Epistolce. Pars altera, Saumur, D. de Lerpinière et J. Lesnier, 1665. Les epistolce adressées à Bouhéreau sont les suivantes : XII, XVII, XVIII, XX, XXIX, XXXI, XXXII, XXXIII, XXXV, XXXVI, XL, XLI, XLVI, LVIII, LXII, LXIII, LXIV, LXVI, LXVIII, LXXI, LXXII. 
permettait de tisser et de maintenir, et l'amitié parfois intense qu'elle leur donnait l'occasion d'exprimer.

Pour entretenir une correspondance avec plus de cent cinquante correspondants il fallait avoir d'importants moyens financiers, étant donné le montant du port des lettres à cette époque. Or Élie Bouhéreau, né en 1643, était le fils unique d'un pasteur qui portait le même nom que son fils - il avait été nommé d'abord à Fontenay et ensuite à La Rochelle - et de Blandine Richard qui, elle, était d'une famille de marchands, semble-t-il, de l'île de Ré. On sait combien modeste était la rémunération des pasteurs à cette époque, dont le salaire était assuré par une taxe spéciale, calculée sur la base de la taille et prélevée par l'église locale ${ }^{8}$. Mais, pour comble de malheur, Élie Bouhéreau père est mort quand le futur épistolier n'avait que dix ans, laissant son fils à la charge de sa veuve. D'où venait donc l'indéniable aisance de cette veuve de pasteur ? Blandine Richard disposait de marais salants dans l'île de Ré, qu'elle gérait avec l'aide de ses deux frères domiciliés à SaintMartin, avec qui elle correspondait. On sait qu'au Grand Siècle le sel était considéré, avec le vin et le blé, comme une des productions fondamentales de l'économie française ${ }^{9}$, et la vente du sel de ses marais salants semble avoir été une des principales ressources pécuniaires de la mère de Bouhéreau.

Grâce à ces revenus, le jeune Bouhéreau a pu faire des études à l'Académie de Saumur de 1656 à 1662, où il a achevé ses humanités classiques (latin, grec) et peutêtre aussi sa philosophie (logique, physique et métaphysique) dans l'idée de devenir pasteur à son tour. Au moment où il quitte l'Académie, le 16 juillet 1662, Bouhéreau était âgé de dix-neuf ans - dans l'ancienne France les jeunes gens entraient à l'université à l'âge où aujourd'hui on entre dans l'enseignement secondaire - et «proposant en théologie », c'est-à-dire un jeune homme qui se destinait au ministère pastoral réformé. Mais, pour des raisons sur lesquelles je reviendrai par la suite, le jeune Bouhéreau a renoncé au ministère protestant en 1664 pendant le premier séjour à Paris que sa mère lui avait offert, grâce de nouveau aux revenus provenant des marais salants. Et trois ans plus tard, en 1667, il part de nouveau de La Rochelle pour faire un voyage de cinq mois en Italie, visitant Venise et Rome en compagnie de son cousin Élie Richard, étudiant en médecine, et s'arrêtant en chemin à Orange, où Bouhéreau se fait conférer un diplôme de médecine - diplôme qu'il a sans doute acheté, car il n'a pas fait d'études de

$8 \quad$ É. Labrousse, Pierre Bayle. I : Du pays de Foix à la cité d'Érasme, $2^{\text {nde éd., }}$ Dordrecht / Boston / Lancaster, M. Nijhoff, 1985, p. 13, n. 5.

9 A. Poitrineau, Art. «Sel », dans Fr. Bluche (dir.), Dictionnaire du Grand Siècle, $2^{\mathrm{e}}$ éd., Paris, Fayard, 2005, p. 1436. 
médecine ${ }^{10}$. Au retour de ce voyage, Bouhéreau fait un deuxième séjour à Paris, cette fois-ci de deux mois à peu près. Un mémorandum des dépenses de ce deuxième voyage, établi en partie par la mère et en partie par le fils, révèle que le voyage d'Italie et le deuxième séjour parisien ont coûté à la famille Bouhéreau 3955 livres, ce qui la met effectivement dans les rangs des familles aisées de La Rochelle.

Pourtant une série de lettres écrites par Blandine Richard à son fils pendant son premier séjour parisien nous permet de mesurer avec plus de justesse l'ampleur réelle de l'aisance de la veuve. La mère écrivait tous les jeudis à son fils, le jour où partait la poste pour Paris ; elle écrivait d'ailleurs dans une orthographe phonétique - comme beaucoup de femmes à cette époque - et signait toujours ses lettres : « Votre mère très affectionnée ». Et si elle envoyait des lettres de change à son fils, qui apparemment demandait sans cesse de l'argent, c'était effectivement parce que sa tendresse maternelle pour ce fils unique lui faisait faire des efforts considérables pour toucher de l'argent à droite et à gauche. Le numéraire était rare au $\mathrm{XVII}^{\mathrm{e}}$ siècle, même au sein de ces milieux aisés, où on ne manquait de rien, mais où les dettes étaient souvent réglées en sacs de blés, volailles, vin ou autres denrées - si toutefois les dettes étaient remboursées. Ainsi Blandine Richard rappelle régulièrement à son fils la nécessité d'être économe, de ne pas retirer de l'argent sans le lui signaler, et de ne pas faire de dépenses inutiles. Déjà le 31 décembre 1663, tout au début du premier séjour parisien, elle faisait des remarques à son fils, qui a évidemment été ébloui par les modes parisiennes, lui reprochant le prix élevé qu'il venait de payer pour une nouvelle chemise, et remarquant qu'il n'aurait pas dû faire poser tant de rubans à ses vêtements. Car cette protestante provinciale et pieuse trouvait que les gens ne devaient pas être « tan garni $^{11} \gg$; pourtant sa tendresse pour son fils était telle que dans une lettre du 21 janvier 1664 elle s'inclinait devant le fait accompli.

Élie logeait chez son cousin Guibert, dans l'ancienne rue de la Boucherie, près de la place Maubert ; et sa mère n'était pas contente d'apprendre que le cousin lui faisait payer plus cher sa chambre qu'il ne l'avait fait payer au cousin Cadolière, d'autant plus que la chambre ne comportait pas de cheminée. Le 21 janvier 1664, elle conseillait à son fils de se procurer un « chofepied afin de ne ce couche point avec le froit »; le 31, elle se demandait comment il faisait sécher son linge, s'inquiétant à l'idée qu'il se mettait du linge qui ne fût pas «bien ceche ». Dans la même lettre, elle se réjouissait de l'arrivée de deux de ses camarades d'études à Paris (en l'occurrence, Jacques Richier de Cérisy et Paul Baudry) ajoutant quand même ce conseil : " mes surtout ne sortes point au soir ». Et toujours les soucis d'argent. Vers la fin du mois de janvier 1664, en lui procurant une lettre de change de 500 livres, elle lui faisait la remarque suivante :

10 Voir P. Benedict et P.-O. Léchot, « The library of Élie Bouhéreau », art. cit., p. 166 ; mais contrairement à ce que disent les auteurs, il n'y a pas de trace dans le cursus du jeune Bouhéreau d'études universitaires en médecine.

${ }_{11}$ Comme pour les citations suivantes, on a conservé la graphie originale des épistoliers. 
vous saves bien la pene que j'ay à en avoir. Vous voies bien comme il sant va vite. Enfin vous le mesnageres le mieux qu'il vous cera possible ses [= c'est] plus votre interes que le mien, si je dessire espargne ce nes [= ce n'est] que pour vous le conserve.

Il s'agit évidemment d'une mère, femme et veuve de pasteur, qui a dû apprendre à ménager son bien, mais qui n'était pas devenue avare pour autant; car, on le voit bien, elle ne pouvait refuser quoi que ce soit à ce fils tant chéri, qu'elle encourageait d'ailleurs à profiter de Paris. Dans une lettre du 6 mars 1664, elle l'incitait à prendre des leçons de musique dans cette ville où on trouvait de bons maîtres; elle se réjouissait des rencontres qu'il faisait dans la capitale, surtout avec les pasteurs du temple de Charenton; et elle lui demandait de raconter un peu sa vie parisienne, lui faisant le reproche suivant : «Vous ne me mandes point comman vous passes votre tan par delà ni les belle chose que vous y voies ». Comme toute jeune personne, le fils était apparemment un peu secret avec cette mère qu'il aimait pourtant en retour.

Un commerce de lettres - pour utiliser le terme de l'époque - de l'ampleur de celle de Bouhéreau nécessitait aussi des qualités intellectuelles exceptionnelles, surtout étant donné que la plupart de ses correspondants étaient eux aussi des hommes fort instruits. Il semblerait que Bouhéreau ait fait de brillantes études à Saumur, à en juger par l'estime qu'avaient à son égard non seulement ses maîtres, Étienne Gaussen ${ }^{12}$ et Tanneguy Le Fèvre ${ }^{13}$, mais aussi ses condisciples. Gaussen, professeur de philosophie à l'Académie, le lui rappelait le 24 mars 1665 :

Mais apres tout il n'y a personne qui vous ayme et qui vous estime comme moy, je n'excuse pas mesme Monsieur Le Fevre quoy qu'il vous cherisse toujours tendrement.

Rappelons-nous que Tanneguy Le Fèvre adressait certaines de ses epistolce latines à Bouhéreau, dont l'une exprimait, écrit Turon de Beyrie le 29 août 1665, « la tendresse qu'il a pour toy et l'estime qu'il fait de ton merite ${ }^{14} »$. D'ailleurs Turon de Beyrie, cet ancien camarade d'études resté ami, n'était pas le seul à demander des conseils de lecture à Bouhéreau, ou à lui poser des questions sur différents livres

12 É. Gaussen (mort en 1675) fut nommé professeur de philosophie à l'Académie de Saumur probablement en 1661, ensuite professeur de théologie déjà en 1664 et agréé par le synode provincial d'Anjou l'année suivante : voir Fr. Laplanche, L'Écriture, le sacré et l'histoire. Érudits et politiques protestants devant la Bible en France au XVII siècle, Amsterdam, APA Holland University Press, 1986, p. 532-545.

13 T. Le Fèvre (1615-1672), régent (1651-1665) puis professeur de grec (1665) à l'Académie de Saumur. Helléniste de renom et philologue, il publia des éditions d'auteurs latins et des traductions en français d'auteurs grecs. Voir Laplanche, L'Écriture, le sacré et l'histoire, p. 545-550.

14 Il s'agit de l'epistola LXXII, T. Fabri Epistolae, éd. cit., p. 346-352. 
ou sur des passages obscurs en latin ; d'autres le faisaient aussi, et tous s'extasiaient sur la « netteté », la « justesse » et l'" agrément » des opinions du Rochelais, le félicitant de son éloquence et accueillant avec joie les poésies galantes qu'il leur envoyait de temps en temps avec ses lettres ${ }^{15}$. Le 16 septembre 1662 , Richier de Cerisy se félicitait d'avoir fait à Saumur la connaissance de Bouhéreau, cet homme « qui a tant d'esprit, tant de douceur, tant de bonté, tant de sagesse, tant de jugement, etc. ». De ces lettres émerge donc la figure d'un jeune homme non seulement fort instruit, mais aussi très spirituel que l'on avait beaucoup de plaisir à fréquenter. Pour ces réseaux protestants de province, le jeune Rochelais était tout ce qu'il y avait de plus accompli selon le modèle de l'époque ; et le professeur Gaussen n'était pas le seul à le remarquer dans une lettre non datée, écrivant à son correspondant : «Vous êtes toujours le plus honneste homme que je connoisse. » Le particularisme, comme on sait, était une tare sous le règne du Roi-Soleil, mais, contrairement à ce que l'on pourrait imaginer, le protestantisme de ces milieux aisés et cultivés s'accommodait sans trop de peine à l'art de vivre et à l'urbanité ambiants que l'on dénommait honnêteté.

Mais Bouhéreau avait aussi d'autres atouts majeurs : il avait eu la chance, et bien sûr les moyens, de faire deux séjours à Paris, cette ville qu'on considérait à l'époque comme étant " la source des belles choses », de l'avis de Turon de Beyrie (7 avril 1665). Le 29 mars 1664, Étienne Gaussen s'impatientait, ayant entendu que son ami rochelais passerait par Saumur dans trois mois pour regagner sa ville natale : «Je voudrois de tout mon coeur que ce fût dans cinq semaines [...] pour nous depaïser de la province, car nous sommes furieusement provinciaux à Saumur. » Si provinciaux, en fait, que l'année précédente on avait demandé au cousin de Bouhéreau, le jeune Élie Richard, étudiant en médecine, d'aller présenter les vœux de la faculté au philologue Daniel Heinsius à Leyde, sans savoir que celuici était mort huit années auparavant. Et Élie Richard de se gausser dans une lettre à son cousin (2 septembre 1664) du « peu de commerce» qu'il y avait entre Saumur et Leyde. Par conséquent, les correspondants de Bouhéreau étaient avides de savoir les nouvelles de la capitale, de la cour, et des modes parisiennes. Le 13 mai 1664, Turon de Beyrie le remerciait d'avoir envoyé avec sa lettre « le dessein du Carrousel de Versailles ». Le jeune Rochelais avait-il été ébloui lui aussi par la fête des Plaisirs de l'Île enchantée que le Roi donnait à Versailles du 6 au 13 mai ? Sans doute, mais son correspondant l'était un peu moins, car il enchaînait avec une remarque amère sur les mœurs du temps : «C'est tout le fruit que la paix produit, et elle ne nous donne que l'oysiveté, le luxe et libertinage dans lequel les hommes et les femmes se perdent egalement. » Le 18 janvier 1668, Étienne Gaussen lui demandait des renseignements sur la mode : " je voudrois me faire habiller à la façon des abbés de Paris ». Le cousin Élie Richard, qui lui aussi eut la chance de faire un séjour dans la capitale, racontait au cousin retourné à La Rochelle la condamnation de Fouquet, Bussy-Rabutin emprisonné à la Bastille et la mort 
d'Anne d'Autriche. Pourtant, ces hommes instruits était aussi et surtout avides des « nouveautés » dans le monde du livre, et voulaient se procurer non seulement les ouvrages savants ou de controverse religieuse récemment parus, mais aussi la toute dernière nouveauté littéraire : La Fontaine, La Rochefoucauld, Molière, BussyRabutin, Racine, Pascal (Les Provinciales), etc. Bouhéreau faisait lui-même ses emplettes, courant les librairies de la rue Saint-Jacques, de la galerie mercière du Palais de justice dans l'île de la Cité et de la place du Puits-Certain dans l'Université, à la recherche aussi bien de livres nouveaux que de livres anciens et d'occasion, pour compléter la bibliothèque qu'il avait apparemment héritée de son père. Et il s'y rendait aussi avec les listes des livres recherchés par ses amis de province pour ensuite les renseigner sur le prix des livres nouveaux, ou la condition d'un livre ancien, avant de les acheter à la demande de son correspondant, et de les faire expédier par le messager avec un billet précisant la somme dépensée qu'on lui rembourserait par des lettres de change ou bien par l'intermédiaire des amis protestants de la capitale, surtout les amis du temple de Charenton.

Il est probable que c'est aussi au temple de Charenton, auquel Bouhéreau se rendait en bateau le dimanche matin tôt avec ses amis de la capitale, qu'il a pu faire la connaissance de Valentin Conrart, premier secrétaire de l'Académie française, et lui-même protestant. De toute façon l'académicien tenait Bouhéreau en grande estime ; c'est l'impression que donne une lettre du 26 mars 1665, envoyée de Paris par Élie Richard au cousin rochelais qui avait regagné sa ville natale. Il raconte par le menu sa propre visite au savant académicien, chez qui il a pu s'introduire parce qu'il apportait une lettre de la part de Bouhéreau, dont " les belles qualités » formèrent par conséquent le sujet principal de l'entretien qui s'est ensuivi. Conrart voulant savoir comment Bouhéreau passait son temps à La Rochelle, Richard lui expliqua que «[son] estude faisoit [sa] principale occupation » et que, pour se détendre, son cousin lisait la poésie française ou bien se divertissait à écrire des vers à quelques-uns de ses amis. Cette réponse provoqua une réaction enthousiaste de la part du célèbre académicien :

Ha, je ne sçavois pas que M. Bouhereau fist des vers, si vous en avies Mr de sa façon vous m'obligeries de me les montrer, je lui dis qu'il n'y avoit pas long temps que vous m'avies envoié une ode que vous avies escrit à un ami en lui envoiant une rose au commencement de l'hyver, et que je ne sçavois pas si je ne l'aurois point sur moy, je cherchai dans deux ou trois poches quoy que je sçeusse bien où elle estoit, à la fin je la trouvai et lui montrai, il la prit et la leut si posement que vous ne sçauries croire, à chaque sixain il disoit, o que voila qui est beau cela est bellissime, quand il eut achevé il dit qu'il reconnoissoit bien là dedans que vous avies leu les poetes anciens, que cela sentoit son Anacreon, et qu'il y avoit là dedans un tour que peu de gens sçavoient prendre. Au reste, j'ay trouvé en lui ce que vous m'avies faict esperer, l'homme du monde le plus civil et le plus obligeant.

Les cousins agissaient-t-ils de concert pour attirer l'attention du grand homme sur les talents littéraires du Rochelais ? C'est fort probable. Quelques années plus 
tard, le 5 juillet 1669, Turon de Beyrie se réjouissait de l'intérêt que portait Conrart au développement intellectuel de Bouhéreau, à qui l'académicien avait proposé de faire une traduction en français du traité d'Origène contre Celse.

Je suis bien aise de voir que tu te caches inutilement et que tout ce qu'il y a de plus éclairé dans le Royaume veuille à toute force te mettre dans le grand jour. Que je veux de bien à $\mathrm{M}$. Conrart de te donner petit à petit matiere d'occupation et de te faire connoistre. J'eusse seulement souhaitté qu'il l'eust mieux choisie, parce que je croy le travail qu'il te propose fort grand et un peu ingrat. Tu me ferois une infidelité horrible si tu ne me communiquois tout ce que tu feras là dessus.

Bouhéreau ne devait publier cette traduction qu'en 1700, vingt-cinq ans après la mort de Conrart, mais celui-ci continuait à jouer son rôle de mentor auprès du jeune Rochelais, qu'il inviterait dans les années 1670 à participer à la révision des Psaumes en vers français. C'est peut-être par l'intermédiaire de Conrart ou bien de Tanneguy Le Fèvre, que Bouhéreau a pu aussi pénétrer dans le cercle de Gilles Ménage à Paris tout au début de son premier séjour parisien. Dès la fin du mois de janvier 1664, Turon de Beyrie le félicitait de cette chance, lui signalant qu'il voulait savoir « jusques aus moindres particularités de ce qui se passera dans vos sçavantes conversations ». Etait-ce la fréquentation de ces cercles mondains et galants à Paris qui avait détourné Bouhéreau de ses études en théologie?

Le jeune protestant, tout fraîchement débarqué à Paris de sa province - sans doute un peu étroite - a certainement été ébloui par ce monde des lettres qu'il découvrait dans la capitale, notamment par « ce Paris des huguenots libéraux », pour reprendre une expression de Roger Zuber, qui maintenait « la présence réformée dans les milieux savants et aisés de la capitale ${ }^{16} »$. Avec eux il découvrait le théâtre, puisqu'ils avaient organisé ensemble « une partie de comédie » qui avait coûté à chacun deux louis d'or : seul Bouhéreau, paraît-il, pour qui cette expérience était évidemment sans précédent, ne regrettait pas son argent ${ }^{17}$. Il s'agissait peut-être d'une pièce de Molière que l'on donnait au mois de janvier 1664, à laquelle il est fait allusion dans une des lettres ${ }^{18}$. Voilà une activité qui l'aurait incité à être un peu secret devant la huguenote pieuse et provinciale qu'était sa mère. En fréquentant ces huguenots libéraux, et surtout les cercles de Conrart et de Ménage, ces mentors et maîtres parisiens du goût qui tous deux s'exerçaient volontiers à la galanterie, avaitil commencé à vivre de façon plus détendue ? Rappelons-nous que le jeune homme avait très vite ajusté ses vêtements aux nouvelles modes enrubannées de Paris, provoquant les griefs de sa mère. Cette manifestation vestimentaire de

16 R. Zuber, « Entre Paris et La Haye : Frémont d'Ablancourt (1621-1693) réfugié », dans O. Elyada et J. Le Brun (éd.), Conflits politiques et controverses religieuses. Essais d'histoire européenne aux $16^{e}-18^{e}$ siècles, Paris, EHESS, 2002, p. 223-224.

17 De Richier de Cerisy, 23 juin 1664 ; Richier n'apprenait que depuis peu, de la part d'un tiers, la nouvelle de cette sortie au théâtre que son ami lui avait cachée.

18 De Turon de Beyrie, 29 janvier 1664. 
consommation frivole était sans doute le signe d'une assimilation à la civilité du paraître et à l'honnêteté urbaine de la capitale. Était-elle aussi le signe d'autres ambitions naissantes, peut-être des ambitions littéraires ? C'est très probable, car étant donné « la coïncidence entre costume et position sociale » sous l'Ancien Régime $^{19}$, ce code vestimentaire mondain était tout ce qu'il y avait de plus incompatible avec une vocation de pasteur. Le jeune homme enrubanné à dû se confier à Turon de Beyrie, car peu de temps après l'arrivée de Bouhéreau à Paris, son ancien camarade d'études est au courant de sa décision d'abandonner la théologie (29 janvier 1664) :

J'approuve fort la resolution que tu as faite de quitter pour quelque temps l'habit et le personage de proposant, abandonne toy entierement aux plaisirs innocens car pour les autres je te les deffens absolument latet anguis in herba [le serpent se cache sous l'herbe], et je fais des vœus que tu ne trouves point d'amertume parmy tous tes divertissements.

Le jeune provincial ayant été séduit par le tourbillon parisien et voulant profiter des nombreux divertissements offerts par la capitale trouvait inconvenante la tenue d'étudiant en théologie et la gravité qui devait l'accompagner. La sémiologie de l'urbanité parisienne et des mœurs mondaines qui se lit dans son habit enrubanné va de pair avec un nouveau centre d'intérêt, peut-être littéraire, mais surtout galant. Comme la remarque de Turon le suggère, Bouhéreau était amoureux d'une jeune fille de bonne famille au nom séduisant de Mademoiselle de Beauchamp, qui allait d'ailleurs lui briser le cœur. Il s'en remettrait pourtant pour se marier au mois de novembre 1668 avec sa cousine, Marguerite Massiot, la belle " Philis » évoquée dans les lettres qu'on lui adressait à cette époque pour le féliciter de son mariage.

Les moyens financiers de Bouhéreau, sa personnalité attachante et spirituelle, ses séjours dans la capitale, son voyage d'Italie, ses talents littéraires, ses contacts parisiens, sa belle bibliothèque, tous ces atouts faisaient de lui un être d'exception dans ces milieux peut-être un peu ternes du protestantisme de province. Il est certain que ses amis enviaient ce qu'Étienne Gaussen appelait avec humour le 24 mars 1668 « ce loisir d'honneste homme dont vous joüissés à La Rochelle », ajoutant : «Quand Mr Choüet ${ }^{20}$ et moy faisons des chasteaux en Espagne, nous vous proposons toûjours comme l'idée parfaitte du bonheur. » Correspondant assidu dont ces amis appréciaient l'« exactitude », c'est-à-dire la fidélité, Bouhéreau mettait ce loisir exceptionnel à profit non seulement pour vaquer à ses études, mais aussi pour rester en contact avec ses amis et coreligionnaires. On oublie l'isolement culturel de

19 Voir D. Roche, La Culture des apparences. Une histoire du vêtement aux XVII et XVIII siècles, $2^{\mathrm{e}}$ éd., Paris, Fayard, 2007, p. 59-60.

20 Jean-Robert Chouet (1642-1731), professeur de philosophie à l'Académie de Saumur de 1664 à 1669, et ensuite à l'Académie de Genève. Voir M. Heyd, Between orthodoxy and the Enlightenment : Jean-Robert Chouet and the introduction of Cartesian science in the Academy of Geneva, La Haye, M. Nijhoff, 1982, p. 24-37. 
ces pasteurs et érudits de province, qui étaient souvent les seuls dans leur entourage à s'intéresser au savoir et aux livres. Son ami Turon de Beyrie le lui rappelle le 3 juin 1663 : « Je suis dans un destroit où il y a autant d'esprit qu'en lieu du monde, mais mon dieu que cest esprit est mal conduit, qu'il est mal tourné et il n'a pas ceste douceur qui est toute particuliere à ceus de la Loire. \Il écrit de nouveau le 29 janvier 1664 : «je suis confiné dans un desert », ou encore le 29 août 1665 : «je suis dans un miserable recoin qui ne porte que de la neige et des frimats » - il habitait en Béarn. Les amis du Rochelais avaient donc des raisons pour s'attacher à ce correspondant fidèle qui avait de quoi alléger leur isolement. Turon de Beyrie, s'apitoyant sur son sort dans cette même lettre du mois d'août, tourne son isolement en un joli compliment pour remercier l'ami rochelais de sa libéralité : « Je suis predestiné à ne pouvoir rien aprendre que par ton moyen, et ma science est plustost l'enfant de ton esprit que celuy du mien. » Le jeu de mots sur la prédestination en dit long sur la haute estime que portaient au Rochelais ses amis de province, et révèle l'importance qu'il avait à leurs yeux dans la circulation des nouvelles et l'échange intellectuel.

Avec ses entrées à Saumur et à Paris, Bouhéreau l'épistolier jouait un rôle d'intermédiaire, transmettant les nouvelles provenant de l'Académie et de la capitale à ses correspondants, envoyant à Paris les manuscrits d'ouvrages rédigés par les amis pasteurs pour avoir l'avis et l'imprimatur de l'académicien Conrart, faisant circuler les nouveautés littéraires et « le sentiment des sçavans ${ }^{21}$ » à leur sujet, prêtant ses livres, et enfin améliorant l'expression française et corrigeant les épreuves des ouvrages de controverse et des sermons que ses amis destinaient à la publication. Somme toute, Bouhéreau jouait le rôle de l'acolyte de Conrart en province, car l'académicien aimait garder le contact avec la jeune génération; par le biais de son correspondant rochelais il pouvait servir de mentor à une nouvelle cohorte d'écrivains et pasteurs protestants. Ainsi, les correspondants du jeune Bouhéreau qui étaient à l'écart des axes de communication et, par conséquent, avides de savoir ce qui se passait dans le monde, écrivaient parfois au Rochelais pour tout simplement solliciter une réponse de sa part. Tel est le cas du pasteur Jean Barin $^{22}$ à Marans (plus tard nommé à l'Académie de Saumur), qui demande le 5 janvier 1673 sa «part des nouvelles que [son] commerce de Paris et de Saumur [lui] aura fournies », et qui écrit de nouveau le 13 décembre 1674 :

Je suis d'ailleurs dans une solitude qui rend mon importunité en quelque sorte excusable. J'ay besoin de quelque consolation, et d'un commerce raisonnable. Ayez

$21 \quad$ De Gaussen, 24 décembre 1667.

22 J. Barin ou Barrin (1630 ? - 1712), pasteur à La Roche-Chalais et à Marans avant d'être nommé à Saumur. Arrêté et emprisonné à La Rochelle en 1685, il se réfugia à Amsterdam où il fut nommé pasteur de la seconde Église wallonne. Il fut rédacteur des Nouvelles de la République des Lettres à partir du mois de septembre 1687 jusqu'à son interruption en avril 1689 : voir É. Labrousse, Pierre Bayle, op. cit., p. 219 n. 
la bonté de souffrir que je le cherche avec vous. Dites moy quelque chose qui me réjouysse. $^{23}$

Et Bouhéreau, en honnête homme parfait et dont on appréciait la "civilité », essayait d'être à la hauteur de tout ce que l'on attendait de lui, prodiguant à ses correspondants «mille témoignages d'amitié », pour reprendre une expression d'Étienne Gaussen de Saumur. Ce thème de l'amitié amène à se pencher sur la pratique et la représentation de la lettre.

La lettre, comme on sait, rend l'absent présent, rappelant au correspondant le souvenir de celui qui écrit. Pour les correspondants du jeune Bouhéreau, la lettre est tout d'abord " une consolation » que l'on recherche pour atténuer la douleur éprouvée devant « l'eloignement des personnes que l'on ayme le plus au monde » - c'est son camarade d'études Richier de Cerisy qui l'écrit le 18 août 1662 après le départ de Bouhéreau de l'Académie. Dans un petit billet non daté Gaussen avoue : « Jamais je ne regarde vôtre étude que vôtre absence ne me touche en l'endroit le plus tendre de mon ame. » Élie Richard, le 11 juillet 1665, envoie lui aussi un billet à son cousin pour lui « souhaiter le bon soir sans [1'] entretenir le moins du monde », finissant sa missive sans cérémonie avec ces mots : «Je m'endors. » L'ami Pierre Meherenc, sieur de La Conseillère ${ }^{24}$, au moment de quitter Saumur pour Sedan lui adresse le 23 mai 1665 un petit mot pour exprimer « le desplaisir qu['il] aura[it] de [s']esloigner de [lui] », ajoutant : « Je ne seray pas tout à fait malheureux si je suis un peu dans vôtre esprit. » Et la réaction des correspondants quand ils recevaient une lettre de la part du Rochelais en dit long sur l'importance qu'ils accordaient à cette présence amicale dont la lettre était le signe. Ayant reçu trois missives au même moment, Turon de Beyrie évoque, le 29 janvier 1664, le « torrent de volupté » déclenché par leur arrivée, dans lequel, dit-il, "j'ay noyé tout mon chagrin ». Et quand les lettres de leur correspondant rochelais tardaient à venir, le chagrin reprenait le dessus. Paul Baudry ${ }^{25}$, autre camarade d'études resté à Saumur et qui devait bientôt rentrer à Rouen, n'ayant point reçu de nouvelles depuis un moment, s'avouait abattu (5 août 1662) :

23 Voir aussi de Benjamin de Daillon, 30 novembre 1674.

24 P. Meherenc de La Conseillère (1645-1699), pasteur à Alençon, se réfugia en Allemagne dès 1681 , où il fonda une église réformée française à Altona (tout près de Hambourg). Voir É. Labrousse, Inventaire critique de la correspondance de Pierre Bayle, Paris, Vrin, 1961, p. 368. Bouhéreau a sans doute noué ou renoué le contact avec La Conseillère en juin 1664 pendant son passage à Saumur lorsqu'il retournait à La Rochelle.

25 Sur Baudry, voir J. A. Kleinstuber, "The letters of Paul Bauldry and Elie Bouhéreau », Lias, nº 22-1, 1995, p. 119-146. 
Je suis privé de toute la joye dont je suis capable maintenant. Sans mentir, la fortune m'est bien cruelle de m'avoir osté un ami que je cherissois fort tendrement, et de m'empescher à cette heure d'apprendre ce qu'il fait et s'il m'aime.

Pour le réseau des correspondants du jeune Bouhéreau, la lettre était avant tout le signe matériel d'un rapport affectif, elle servait à garder ce contact affectueux, voire à le maintenir, en rendant présent l'ami chéri que l'on ne voyait plus pour l'instant. Tout en n'étant qu'un "piètre substitut d'une présence rêvée », pour reprendre une expression d'Isabelle Landy-Houillon, la lettre servait néanmoins à « combler textuellement les vides de l'absence ${ }^{26} »$. La réception d'une missive inscrivait le rapport interrompu dans le hic et nunc du correspondant, ravivant dans l'esprit et le cœur l'épaisseur d'un vécu partagé que l'on essayait de prolonger dans le présent par le biais de la lettre ${ }^{27}$.

Les allusions ici et là dans cette correspondance à des épîtres en latin, à des lettres badines et galantes ou bien à des missives en vers qui ne sont pas arrivées jusqu'à indiquent qu'Élie Bouhéreau, ce jeune homme accompli, s'entraînait à l'art épistolaire. Paul Baudry, l'ami de Saumur et ensuite de Rouen, lui fait souvent des compliments, s'exclamant le 29 juillet 1662, à la réception de la lettre la plus récente :

Je meure, elle m'a ravi, et je l'ay trouvée admirablement bien faite, soit pour le naturel des pensées, soit pour l'art que vous avés observé dans la disposition de vos mots.

Face à cet homme accompli, les correspondants s'excusaient à mainte reprise du « désordre » des lettres qu'ils lui envoyaient, de la «précipitation » avec laquelle ils écrivaient, ou bien de la " négligence » de leur plume. Tantôt c'était parce que le correspondant voulait courir à la poste, tantôt parce qu'il avait beaucoup d'affaires sur les bras, parce qu'il fallait arrêter d'écrire quand « la clochette appelait à souper », parce qu'un ami venait d'entrer dans le «cabinet » ou tout simplement parce que « le froid empeschoit de tenir la plume ${ }^{28} »$. Il s'agit évidemment d'un laisser-aller bien réel, et non pas de cette esthétique de la négligence que cultivaient les beaux esprits de l'époque qui voulaient simuler dans l'écrit les rythmes de l'échange oral.

26 I. Landry-Houillon, « Lettre et oralité », dans B. Bray et C. Strosetzki (éd.), Art de la lettre, art de la conversation à l'époque classique en France, Paris, Klincksieck, 1995, p. 83.

27 Voir à ce propos, la lettre de Baudry, 30 septembre 1662 : «O que ce n'est pas là ce que je m'estois promis, quand en m'esloignant de vous je pensois aux choses qui pourroyent me faire supporter vostre absence, et que je me figurois bien un autre commerce ; $j$ 'auray de ses lettres du moins une fois chaque semaine, disois-je en moy mesme, et lors qu'il m'aura parlé du progrés de ses estudes et de sa santé, il m'assurera que je seray toujours de ses amis, et qu'il ne changera jamais pour moy ».

28 De Gaussen, 26 octobre 1663 ; de Turon de Beyrie, 5 février 1664. 
D'ailleurs les correspondants envoyaient des lettres rédigées dans la plus grande et la plus réelle spontanéité parce qu'ils savaient que c'était ce que le Rochelais aimait le plus. Élie Richard le lui rappelle dans une lettre du $1^{\text {er }}$ février 1664 :

Souvenés vous que vous m'avés mandé de vous entretenir de tout ce qui me viendroit en la pensée et ne vous estonnés pas si vous voyez peu d'ordre en cette lettre.

Il semblerait que la spontanéité de l'expression, et d'ailleurs de l'émotion, ait été la règle d'or des correspondants. Dans une lettre du 10 septembre 1662, Richier de Cerisy s'en réjouissait :

Vous voyez, mon tres cher Monsieur, que j'en use avec autant de liberté que vous m'en donnez; vous me faites plaisir de m'assurer que vous n'estes point homme de ceremonie et que vous aymez la liberté. Je suis ravi que nous ayons mesme amitié et mesme aversion.

Pourtant ce refus de toute cérémonie, ainsi que de la rhétorique de la civilité par laquelle elle s'exprime, pour réel et spontané qu'il soit, fait partie d'une topique de l'amitié qui remonte à l'Antiquité ${ }^{29}$ et selon laquelle les amis façonnaient plus ou moins consciemment leurs échanges. Pour le réseau de Bouhéreau, les pirouettes de la civilité mondaine se ramènent à la flatterie, qu'ils sont unanimes à récuser ${ }^{30}$. Le cousin Richard avoue ha_r « la flatterie », qu'il croit "indigne d'un honnête homme » ; Paul Baudry sait qu'être flatteur risque de lui ôter « le rang que je tiens parmi vos amis ; ce qui seroit pour moi le plus grand de tous les malheurs ${ }^{31} »$. De l'avis de ces jeunes gens, la négligence stylistique est apparemment le signe d'une intimité supérieure ${ }^{32}$. C'est Turon de Beyrie qui l'exprime dans une lettre du 5 juillet 1669 :

Je suis bien aise que ma negligence ne te bute pas, et elle te doit asseurement faire connoistre que mon cœur est tout entier dans cet entretien et que ton merite et tes bons offices te l'ont extremement attaché.

29 Voir Cicéron, De amicitia, XXIV-XXVI, 88-99. Voir aussi J.-C. Fraisse, Philia : la notion d'amitié dans la philosophie antique, Paris, Vrin, 1974, p. 409-412.

30 Le thème est présent également dans la correspondance de Pierre Bayle, voir R. Whelan, " "Un long commerce" : l'amitié dans la correspondance du jeune Bayle », dans C. Pailhès et alii (éd.), Le Rayonnement de Bayle, Oxford, The Voltaire Foundation, 2010, p. $122-123$.

31 D’Élie Richard, 16 décembre 1663 ; de Baudry, 29 juillet 1662.

32 J'emprunte cette idée ainsi que l'expression à U. Michalowsky, « Malherbe épistolier », dans Art de la lettre, art de la conversation à l'époque classique en France, op. cit., p. 206. 
Pour ces jeunes hommes, le style négligé allait de pair avec l'authenticité de l'amitié : il exprimait mieux que les compliments mondains la sincérité des attaches qui les liaient les uns aux autres et qu'ils tenaient à s'exprimer par le biais de la correspondance.

Ainsi, très souvent, les correspondants se livrent à cœur ouvert, parlant de leurs amours, de leurs soucis d'argent, de leurs maladies, de leurs problèmes de famille, de leurs déceptions et de leurs deuils. Le 19 janvier 1664, Étienne Gaussen écrit sous le choc de la mort survenue la veille de Moïse Amyraut ${ }^{33}$, le célèbre professeur de Saumur et son cher maître, avouant à son ami :

Icy nous sommes en un étonnement [c'est-à-dire un bouleversement] que je ne puis vous exprimer [...]. Je vous écris avec un certain abbatement de cœur que je n'avois point encore senti de ma vie.

La lettre véhicule ici le paradoxe de la communication émotionnelle, qui s'exprime pour ainsi dire par le silence, par le simple geste d'écrire lorsque le langage fait défaut à celui qui veut partager sa peine avec l'ami chéri. Tout porte à croire que les lettres du Rochelais respiraient aussi cette franchise affective, car dans l'été 1668 il se serait livré à son ami Turon de Beyrie, confessant l'amour qu'il ressentait à l'égard de celle qui allait bientôt devenir sa femme et exprimant peut-être aussi par la même occasion les doutes qui le rongeaient à ce sujet. Et l'ami de répondre le 24 juillet 1668 avec cette exhortation :

Hâte toy de posseder la personne qui te semble devoir faire le bonheur de ta vie, et de goûter entre ses bras les plaisirs qui ne nous fuient tousjours que trop et qui ne te produiront jamais les mauvaises suites et l'amertume que tu peux en apprehender.

Le tutoiement réciproque adopté par les deux amis, et qui est exceptionnel dans cette correspondance, est le signe d'une relation épistolaire qui prolonge par l'écrit les confidences que les jeunes gens avaient sans doute l'habitude de se faire de vive voix. Les petites nouvelles banales échangées, les secrets et craintes confiés et reçus, les conseils aussi, font de ces lettres une conversation entre amis, ou plutôt, une causerie, pour reprendre le mot privilégié par Étienne Gaussen, qui écrivait un jour à son ami rochelais :

Il n'est rien qui me délaisse [c'est-à-dire qui me détend] si fort l'esprit de mes speculations metaphysiques que la pensée de vous ouvrir mon cœur et de causer avec vous sans mystere et sans façon.

33 Moïse Amyraut (1596-1664), pasteur et théologien réformé, professeur à l'Académie à partir de 1633. Voir Fr. Laplanche, op. cit., p. 346-353, 379-383, 386-411, 430-470, 475500 et 508-512. 
Une causerie par lettres : le mot en dit long sur tout ce qu'il y avait de familier, voire d'intime, dans ces échanges épistolaires qui comptaient sur la compréhension du correspondant, sur sa sympathie aussi, et enfin sur sa discrétion.

Mais une autre métaphore de la lettre revient incessamment sous la plume de ces protestants lettrés, celle de l'entretien, métaphore qui n'a rien d'original, comme on sait. Selon Bernard Beugnot, « l'entretien est attention à la personne et confie à l'échange le soin d'exposer et de découvrir l'idée ${ }^{34} »$. Ainsi les correspondants aiment prolonger par écrit l'échange d'idées que certains d'entre eux avaient connu à Saumur. Ayant quitté l'Académie pour retourner à Pau, Turon de Beyrie demande au Rochelais de lui inspirer « cette passion qu['il a] pour l'estude », et de lui faire des leçons sur des passages difficiles d'Horace (3 juin 1663). Étienne Gaussen lui envoie au mois de septembre de la même année un exemplaire de ses thèses de philosophie, attirant l'attention de son ami sur « la maniere dont [il s'est] advisé d'accorder en plusieurs choses Épicure et Descartes avec notre Aristote ». Avec Paul Baudry à Rouen, Bouhéreau se livre à un travail de critique minutieuse sur des textes anciens, tant sacrés que profanes ; et, le 2 novembre 1663, Baudry incite son ami à reprendre dans ses lettres les joutes oratoires qu'ils avaient sans doute connues à Saumur :

Vous estes admirable en verité de ne vouloir pas soûtenir une opinion contre moy. Et pensés-vous que je vous sçache mauvais gré quand vous rejettés les miennes ? Point du tout je vous jure ; et n'en approuvassiés-vous jamais aucune, je ne vous en estimeray jamais moins, et jamais je ne vous en aimeray point. S'il se trouve donc que je vous en propose qui ne vous puissent agréer, choqués-les, renversés-les s'il vous est possible. Car si vous le faites avec raison, je suis fort trompé si enfin je ne vous cede à la fin. Et si je pense que vous vous mépreniés, il ne vous en coustera rien de l'ardente amitié que j'ay pour vous.

Pour ces jeunes gens fraîchement sortis de l'Académie, la lettre peut devenir le lieu d'un dialogue intellectuel, voire d'un débat : elle inscrit l'autre dans le texte de la missive, non comme un autre soi-même, mais plutôt comme l'autre en face de $\mathrm{soi}^{35}$. Comment expliquer la réticence de Bouhéreau à l'égard du débat sollicité ? Elle est peut-être indicative d'une autre conception de l'échange qui, refusant les joutes oratoires in utramque partem, privilégie plutôt l'ingenuitas, c'est-à-dire la liberté d'une parole qui peut s'exprimer sans crainte et sans flatterie, mais surtout sans avoir recours aux fariboles de la rhétorique ${ }^{36}$. Peut-être pour le Rochelais, la

34 B. Beugnot, « L'entretien », La Mémoire du texte. Essais de poétique classique, Paris, H. Champion, 1994, p. 154.

35 Même phénomène chez le jeune Bayle : voir R. Whelan, «"Un long commerce" : l'amitié dans la correspondance du jeune Bayle », art. cit., p. 123-124.

36 Sur cette notion de l'ingenuitas, voir S. J. Savonius, « The role of Huguenot tutors in John Locke's programme of social reform », dans A. Dunan-Page (dir.), The Religious Culture of the Huguenots (1660-1750), Aldershot, Ashgate, 2006, p. 150-152. 
lettre, autant que l'amitié qu'elle véhiculait, offrait une sphère de liberté où les amis pouvaient vaquer à la découverte partagée mais désintéressée de la vérité. Elle devait faciliter une rencontre authentique entre deux êtres intelligents qui aboutirait à une valorisation de l'autre, mais tout en établissant une distance entre soi-même et l'autre. La lettre, à son sens, devait créer un espace commun où les " honnêtes hommes » pourraient s'entretenir avec cette franchise prévenante qui seule permet de devenir soi-même avec l'autre dans un vis-à-vis qui fait avancer ${ }^{37}$.

Dans cet espace libre de découverte et de partage tous les correspondants s'entretiennent par écrit des livres qu'ils lisent, exprimant leur opinion là-dessus et s'attendant que l'autre fasse de même. Quand au printemps 1665, Gaussen envoie de nouveau des thèses, cette fois-ci de théologie, il demande à Bouhéreau de les lire et « de [lui] dire [son] advis comme on le dit à un amy, c'est à dire, fortement et librement ». Et cette liberté dans l'échange intellectuel faisait tout leur bonheur. Turon de Beyrie, revenu en Béarn après un petit séjour à La Rochelle, essaie de prolonger un peu de cette ambiance dans et par sa lettre du 10 juillet 1668 :

$\mathrm{Tu}$ vois par la maniere dont je t'escris que mon cœur veut t'entretenir dans mes lettres avec le mesme abandonnement et le mesme desordre que dans ces courts et bienheureux moments que j'ay eu le bien de t'entretenir dans ton cabinet.

Pour tous ces correspondants, la lettre savante fonctionnait comme une sorte de cabinet virtuel, cet espace où on pouvait se retrouver dans l'intimité ${ }^{38}$, sans doute entre hommes, pour parler de tout ce qui leur passait par la tête. C'était un espace auquel Bouhéreau était très attaché, et dont il défendait l'entrée à tous ceux qui n'étaient pas invités ; car pendant son absence à Paris, sa mère tenait à le rassurer : «Personne n'antrera point en votre cabinet ne vous en metes point en pene » (14 janvier 1664). Pourtant cet espace intime était aussi ouvert sur le monde, puisque là l'épistolier aimait retrouver ses amis non seulement réels, mais aussi virtuels, qu'il s'agît de ses correspondants, ou bien de ses livres. À son retour de Paris en 1668, Étienne Gaussen demande à Bouhéreau des nouvelles de « Mrs [ses] livres» : « Mandés moy si Mrs vos livres se portent bien et si vous vous estes réconciliés ensemble de bonne foy » (24 mars 1668). Le cabinet, les livres et la correspondance permettaient au Rochelais de vivre sur le plan réel, et de revivre, sur le plan imaginaire, « ces tranquilles plaisirs et ces entretiens innocents qui rendent une vie douce et heureuse ", pour citer de nouveau son ami Turon de Beyrie (10 juin 1664). D'espace de recueillement, le cabinet - et la lettre qui le recrée devient un lieu de rencontre et de fréquentation, tantôt réelles, tantôt rêvées, entre hommes du même esprit.

37 S. Requemora, «L'amitié dans les Maximes de La Rochefoucauld », XVII siècle, $\mathrm{n}^{\circ} 205$, octobre-décembre 1999, p. 702.

38 Voir O. Ranum, "Les refuges de l'intimité », dans R. Chartier (éd.), Histoire de la vie privée. III : De la Renaissance aux Lumières, $2^{\mathrm{e}}$ éd., Paris, Éditions du Seuil, 1999, p. 227-228. 
Pourtant dans le cabinet virtuel que ces jeunes huguenots recréaient par le biais de leurs lettres, le ton n'était pas toujours aussi sérieux. Sachant que leur ami de La Rochelle était « fort homme d'esprit », pour reprendre une expression de Richier de Cérisy, et qu'il aimait autant rire qu'un autre, ils tentaient d'égayer de boutades, de badinage, de « bagatelles » et d'histoires drôles leurs entretiens par lettres. Le 23 mai 1665, Pierre Meherenc, sieur de La Conseillère, voulant établir « un commerce de lettres » avec le Rochelais, regrette de ne pas pouvoir l'entamer avec, dit-il, « quelque chose de divertissant et de nouveau pour debuter bien, je veux dire pour commencer agreablement un commerce dont je souhaite ardemment la continuation ». Dans une lettre datée du 30 juin 1664, Richier de Cérisy, voulant consoler son ami qui venait de quitter Paris pour La Rochelle, lui raconte l'humeur folâtre qui s'était emparée des jeunes gens dans le bateau qui les amenait au temple de Charenton. Ils lorgnaient les filles. Surtout Mademoiselle Dumoulin, « qui vaut bien 50 personnes » : «nous vismes 2 ou 3 fois ses mains et ses bras et une gorge [c'est-à-dire son décolleté], qu'on voyoit assez au travers d'un petit mouchoir pour juger qu'elle estoit extremement belle ». Et Richier de rappeler à son correspondant le plaisir qu'il aurait pris à voir cette belle personne et à faire le galant. Quoique sans doute fort pieux, ces jeunes protestants ne se refusaient pas non plus le plaisir de la raillerie aux dépens de protestants d'une tradition différente de la leur. Élie Richard voulant partager avec le cousin rochelais les « raretés » de la Hollande où il faisait ses études, lui raconte "la simplicité des Anabaptistes » (16 décembre 1663) :

pas un n'est plus brave que l'autre, ils ont les cheveux coupés jusques aux oreilles avec des colets de Jesuites ; chacun d'eux donne selon ses richesses et ne cachent point ce qu'ils ont, celui d'entre les docteurs qui se leve le premier et qui se dit inspiré, est celui qui presche, aucun ne prie Dieu haut mais chacun à part soy, ils jettent des souspirs si lamentables qu'ils vous feroient crever de rire.

Pour provoquer ce fou rire, qui les consolait de la distance qui les séparait, les amis envoyaient aussi des gazettes burlesques, ou bien se moquaient volontiers d'eux-mêmes et parfois affectueusement des autres, s'en remettant au tact de leur destinataire à qui ils demandaient de veiller à ce qu'aucun écho de leurs indiscrétions ne parvienne aux oreilles de ceux qui n'étaient pas censés les savoir. Le cousin Élie Richard, s'inquiétant un jour à ce sujet, rappelle au Rochelais que les lettres qui portaient en tête un petit signe contenaient des passages écrits à l'encre sympathique, et qu'il suffirait de tremper sa lettre dans l'eau pour faire apparaître ces remarques compromettantes ( $1^{\text {er }}$ février 1664). Évidemment, les correspondants faisaient circuler lettres, extraits de lettres, ou nouvelles autour d'eux, car à l'époque la distinction entre le privé et le public n'était pas, comme on sait, aussi étanche qu'elle allait devenir par la suite. Pourtant le rire partagé et indiscret délimite à 
l'intérieur de la parole publique un lieu privilégié d'échange intime fondé sur une complicité qui exclut ${ }^{39}$.

Ainsi les lettres échangées entre ces jeunes amis prolongeaient par l'écrit l'ambiance de ce « groupe de convivialité », pour reprendre l'expression de Philippe Ariès $^{40}$, qu'ils avaient forgé à Saumur : groupe de personnes choisies et chéries qui se distingue de la famille d'une part et de la société environnante de l'autre. Les lettres échangées continuaient à véhiculer l'échange intellectuel et le sens de l'humour du groupe, mais elles créaient en même temps un lieu protégé du regard étranger, un lieu d'affectivité où ces jeunes hommes exprimaient librement l'affection, voire l'amour, qui les liaient les uns aux autres. Il ne s'agissait pas uniquement de ces formules de politesse dont les correspondants étaient prodigues à l'âge moderne, et qui faisaient partie des codes de cette " civilité " étudiée par Jacques Revel ${ }^{41}$. Je pense par exemple à des salutations du genre : " Aimez tousjours celuy qui vous aime de tout son cœur »; ou bien : «c'est, je vous jure, le premier de tous mes biens que d'estre aimé de vous »; ou encore : « je suis de tous les humains celui qui vous cherist le plus »; ou enfin : «il n'y a personne qui vous ayme et qui vous estime comme moy ${ }^{42} »$. Ce genre de courbettes amicales se répète dans toutes les correspondances de l'époque et servait à entretenir des relations d'amitié. Pourtant, encadrées par des formules de ce genre, apparaissent des expressions d'une amitié plus intime qui méritent que l'on s'y arrête, car elles contribuent à cette histoire des sensibilités au Grand Siècle - toujours assez mal connue faute de témoignages.

À vrai dire, les rapports affectifs tissés au sein du groupe de convivialité que le jeune Bouhéreau a construit autour de lui, ressemblent plutôt à des relations d'amour qu'à des attaches amicales. Étienne Gaussen, entouré néanmoins à l'Académie de Saumur d'hommes intelligents, aimait rappeler à son ami de La Rochelle qu'en lui il avait trouvé l'homme de sa vie (12 février 1664) :

J'ay bien plusieurs autres amis avec qui $\mathrm{j}$ 'ay quelque commerce, mais vous estes proprement l'homme que je cherchois il y a long-temps, et que j'ay trouvé à la fin pour le plus grand bonheur du monde.

Au mois de novembre 1662, Paul Baudry, demeuré un moment à Saumur après le départ de Bouhéreau, demandait à son ami des témoignages de son affection,

39 Le 29 mars 1664, Gaussen rappelle à son correspondant qu'il ne faut pas communiquer ses lettres à des personnes qui seraient choquées par le franc-parler dont les amis usaient entre eux ; au mois de septembre 1663 il avait déjà prié son ami « de ne faire voir [ses] lettres à qui que ce soit, afin qu['il lui] parle avec plus de liberté ».

40 «Pour une histoire de la vie privée », dans Histoire de la vie privée. III, op. cit., p. 17.

41 J. Revel, «Les usages de la civilité », ibid., p. 167-208.

42 De Richier de Cerisy, 23 septembre 1662 ; de Baudry, 29 juillet 1662 ; d’Élie Richard, 12 mars 1665 ; de Gaussen, 24 mars 1665. 
utilisant - peut-être à son insu, mais peut-être pas - le langage de la sensibilité galante (4-6 novembre 1662) :

Mais parlés moy bien, je vous conjure, de la tendresse que vous avés euë autrefois pour moy et de tous les petits soins qui naissoyent si naturellement de l'amitié que vous m'aviés donnée [...].

La tendresse et les petits soins échangés entre ces jeunes hommes rappellent la Carte de Tendre et les conseils qu'elle contient pour se rendre aimable auprès des femmes. D'ailleurs Baudry n'était pas le seul à avoir recours au registre de l'amour pour exprimer son amitié. Gaussen employait volontiers, lui aussi, ce langage de la galanterie, remarquant dans une des lettres qu'il envoyait à son ami lors de son deuxième séjour parisien (fin novembre 1667):

Jamais amant n'a fait plus de folies en l'absence de sa maitresse que vos voyages m'en ont fait faire. Mr Chouet m'en est un fort bon témoin qui m'a reproché souvent ces hyperboles d'amour qui me sont si ordinaires toutes les fois que je parle de mon cher Mr Bouhereau.

Comment interpréter le langage hyperbolique et les expressions galantes que ces jeunes hommes utilisaient pour dire leur affection (sinon leur amour) au Rochelais, qui leur donnait à son tour des témoignages du même genre ? Le 6 novembre 1662, Paul Baudry remerciait son ami de « la maniere si obligeante dont [il avait] promis de [1']'aimer », et se réjouit d'" avoir toujours place dans [son] ame ». Dans « la gamme de variétés » de l'amitié à l'âge moderne, évoquée par Philippe Ariès, qui va de la fraternité d'armes à la grande amitié ${ }^{43}$, les rapports affectueux entre le Rochelais et ses amis ne sont pas des moins intenses. Or, comme nous le rappelle Orest Ranum, l'amitié intime est à cette époque une forme d'amour ${ }^{44}$. Si les correspondants voulaient se donner des preuves de cette philia, c'est-à-dire de cet amour réciproque et non sensuel entre amis, à quel autre langage auraient-ils pu avoir recours sinon au langage courant de l'amour dont on se servait volontiers à leur époque pour exprimer ses sentiments ? D'ailleurs, quand ils se confiaient leur affection réciproque, ces jeunes provinciaux, tous admirateurs de Conrart, croyaient peut-être se parler selon les modes langagières parisiennes, ignorant que la préciosité n'était plus de mise dans la capitale.

Mais une question demeure. Comment interpréter les sentiments que des jeunes hommes s'exprimaient ainsi et réciproquement ? Turon de Beyrie, qui aimait lui aussi évoquer la tendresse qu'il ressentait à l'égard de son ami rochelais, révèle un jour ce dont il s'agissait (10 juin 1664) :

44 O. Ranum, « Les refuges de l'intimité », art. cit., p. 255. 
Je croirois pecher contre la franchise de nostre amitié si je ne te faisois voir mon ame toute nue et si je ne t'en descouvrois l'agitation. Je suis pourtant un peu interessé dans cette ouverture et je te dis mes pensées afin que tu m'ouvres ton coeur, et je verray par là si le Ciel qui a fait naistre tant d'amitié entre nos deux cœurs n'a point mis aussy de la conformité dans leurs mouvements et dans leurs ressorts.

Ce langage de l'amour renvoie donc à une sympathie mutuelle que des êtres humains ressentent dans leur cœur, mot qui désigne le for intérieur, le centre affectif de la personne. Quoique les effets de cette sympathie soient visibles dans l'élan qui porte ces êtres l'un vers l'autre, suscitant leurs confidences réciproques, sa cause reste inexplicable, puisqu'elle arrive, comme par miracle, du Ciel - ce qui veut dire pour un protestant (ou d'ailleurs un augustinien) par un effet de pure grâce. Mais une fois établie, cette sympathie comporte des obligations, dont la principale est la franchise, cette nudité de l'âme et cette ouverture à l'autre si joliment évoquées par Turon, qui permettent à l'ami de voir la personne telle qu'elle est, et sans façons. L'ingenuitas et la franchise affective recherchées par ces jeunes hommes sembleraient donc renvoyer au modèle sénéquien des Lettres à Lucilius où les correspondants prolongent par l'écrit leur « recherche commune de la vérité à partir d'une mise à jour du propre moi s'analysant », pour reprendre une remarque de Viviane Mellinghoff-Bougerie ${ }^{45}$. Voilà pourquoi, dans cette même lettre, Turon de Beyrie demande au jeune Bouhéreau de l'aimer toujours, et de ne jamais interrompre leur « commerce » épistolaire :

avec toy je suis philosophe. Je me sens eslevé au-dessus des passions.

Le cabinet virtuel qu'est cette correspondance est donc un lieu idéal et - tel l'étude réelle à l'âge moderne - presque exclusivement masculin ${ }^{46}$, qui permettait à des hommes du même esprit de se retrouver entre eux pour s'entretenir de tout ce qui leur passait par la tête. C'est aussi un espace de convivialité et d'intimité, de complicité et de confiance où les amis se livraient à cœur ouvert, s'analysant, s'encourageant, se façonnant réciproquement. Étant donné l'isolement de leur province respective, leur vie d'intellectuels et de protestants dépendait de cette solidarité collective, de ce réseau épistolaire qui les tenait au courant des nouvelles, des modes, des nouveautés dans le monde du livre et de la culture, et, en fin de compte, des menaces qui commençaient à peser de plus en plus sur leur vie collective - ce qui m'amène à ma conclusion.

45 V. Mellinghoff-Bougerie, « Un entretien sans dialogue ? De la correspondance de François de Sales aux Lettres spirituelles de Jean-Pierre de Caussade », dans Art de la lettre. Art de la conversation à l'époque classique en France, op. cit., p. 194.

46 Voir O. Ranum, « Les refuges de l'intimité », art. cit., p. 225 ; voir aussi, dans le même volume, M. Aymard, « Amitié et convivialité », p. 470. 
C'est de propos délibéré que j'ai limité mes remarques en grande partie aux lettres échangées entre Élie Bouhéreau et ses correspondants pendant les années 1660. Car, comme ces lettres le laissent entendre, il s'agit d'un moment exceptionnel non seulement dans la vie du jeune Rochelais mais aussi dans l'histoire du protestantisme français. Le règne personnel venait de commencer, déclenchant, comme on le sait, une explosion de créativité dans les lettres et les arts sous le mécénat d'un jeune roi à la recherche de la gloire. Et ces protestants de province en étaient affectés autant que les autres sujets du royaume, puisqu'on les voit suivre de près dans la mesure du possible tout ce qui arrivait dans la capitale, grâce à leurs différents interlocuteurs parisiens, parmi lesquels se trouvait pendant un moment le jeune Bouhéreau. La tendance était aussi à l'optimisme dans ce Paris des huguenots libéraux, dont l'allégresse se lit dans la sémiologie des vêtements enrubannés, les sorties au théâtre, les visites de monuments tout récemment construits. Même si différentes interventions officielles auprès des protestants du Poitou et de Saintonge ou de La Rochelle donnaient déjà de l'inquiétude à certains correspondants, y compris à la mère de Bouhéreau, on croyait toujours avoir des perspectives d'avenir dans cette France que l'on chérissait tant. Le 13 décembre 1667, Turon de Beyrie était prêt à parier que le nom de Bouhéreau « seroit un jour aussy connu dans la republique des belles lettres, que le sont celuy de Chapelain, de Menage et de ces autres heros qui y tiennent presentement les premiers rangs ». Il ignorait encore, comme d'ailleurs Bouhéreau lui-même, tout ce que cet avenir réservait pour eux, tout ce que la haine du particularisme que nourrissait le roi ferait déclencher contre des sujets qui n'avaient de particulier que le malheur de ne pas être de sa religion. Ils allaient bientôt le découvrir. Déjà dans les années 1670 , les lettres échangées entre les différents correspondants du réseau de Bouhéreau témoignent de leur inquiétude croissante, voire de leur désarroi devant les tracasseries et brimades qui augmentaient. Et le ton de la correspondance change : les années folâtres n'étaient plus, il fallait maintenant faire face à l'orage. Pourtant, les lettres de jeunesse demeurent, livrant leur secret d'une trajectoire brisée, d'espoirs anéantis et de la nostalgie d'un réfugié vieillissant pour des jours révolus. 